\title{
Vitamin-D Status in metabolic syndrome and its correlation with different parameters of metabolic syndrome
}

Authors

\section{Dr Rahul Kumar ${ }^{1}$, Dr Umashankar U S ${ }^{2}$, Dr VPS Punia, Dr B.C. Kabi, Dr Bhandari Gurbir Singh, Dr Harshit Khosla ${ }^{2}$, Dr Akash Jain ${ }^{2}$}

Department of Medicine, Vardhman Mahavir Medical College and Safdarjung Hospital, New Delhi ${ }^{1}$ Assistant Professor, Department of Medicine, Jawahar Lal Nehru Medical College and Hospital, Ajmer, Rajasthan, Pin code-305001

${ }^{2}$ PG Resident, Department of Medicine, VMMC \& Safdarjung Hospital, New Delhi 110029 Corresponding Author

\section{Dr Rahul Kumar}

Room no 96, Medicine Office, Jawahar Lal Nehru Hospital, Ajmer, Rajasthan- 305001

Email:dr.rahulvmmc@gmail.com

\begin{abstract}
Introduction: Metabolic syndrome refers to cluster of several cardio metabolic risk factors including abdominal obesity, hyperglycemia, dyslipidemia and elevated blood pressure that likely linked to insulin resistance. Vitamin-D deficiency may be a risk factor of metabolic syndrome.

Objective: This study was performed to evaluate the role of vitamin-D in patients of metabolic syndrome and find out its correlation with different parameters of metabolic syndrome.

Material and Methods: 150 metabolic syndrome patients and 150 controls were recruited. Waist circumferences, blood pressure, vitamin-D and other parameter of metabolic syndrome (serum triglyceride, serum HDL cholesterol and fasting plasma sugar) were measured in blood sample collected between January 2012 and December 2014.

Results: Metabolic syndrome patients had significantly reduced circulated level of 25-hydroxy vitamin-D compared with the controls. It was seen that $60 \%$ of the cases were deficient in vitamin-D while only 10 $\%$ of the controls were deficient in vitamin D. Cases had higher mean values of systolic and diastolic blood pressure, fasting blood sugars, triglyceride levels and lower mean HDL cholesterol as compared to the controls.

Conclusion: vitamin-D levels were decreased as the metabolic syndrome patients had more than 3 components of metabolic syndrome and showed inverse relationship with increasing number of components.

Keywords: Metabolic syndrome, vitamin D, vitamin-D deficiency.
\end{abstract}

\section{Introduction}

Metabolic syndrome $(\mathrm{MeS})$ is characterized by several abnormalities including impaired glucose tolerance, blood lipid derangements, and prothrombotic and pro-inflammatory states. ${ }^{(1)}$. It is believed that visceral fat, by secreting some inflammatory adipokines, brings about both insulin resistance and an inflammatory state. ${ }^{(2)}$. Adipose tissue has been shown to act as a "metabolic well" for vitamin-D hence reducing its 
bioavailability. ${ }^{(3)}$ Elevated blood inflammatory biomarkers such as C-reactive protein (CRP), interleukins, extra fat deposition in the body, depending on its location, can adversely affect vitamin-D status. ${ }^{(4)}$ The clinical relevance of metabolic syndrome is that it defines people who are at increased long-term risk of cardiovascular and type 2 diabetes mellitus. ${ }^{(5)}$ Accumulating research suggests that circulating concentrations of vitamin-D may be inversely related to the prevalence of diabetes ${ }^{(6-10)}$, the concentration of glucose $^{(8,11,15)}$, and insulin resistance ${ }^{(9,10)}$. Vitamin-D deficiency is related to impairments in insulin secretion and impaired insulin synthesis. Calcium regulates insulin synthesis within pancreatic $\beta$-cells, as well as insulin secretion; and plasma calcium levels are mediated by vitamin $D$. (10-15) Circulating 25 hydroxyvitamin-D (25 $[\mathrm{OH}] \mathrm{D})$ has been inversely associated with abdominal adiposity, hypertriglyceridemia, and hyperglycemia. The immune-modulatory effect of vitamin-D and its inverse link with inflammation draws more attention to its possible role in MeS. Obesity and MeS are usually accompanied by "low-grade systemic inflammation" and augmented oxidative stress, both of which are believed to have pivotal roles in development of $\mathrm{MeS}$ itself and in its further morbidities. Low vitamin-D status has been linked to inflammatory endothelial dysfunction in both diabetic and non diabetic subjects.

\section{Material and Methods}

A total of 150 cases were included in the study satisfying the inclusion criteria, 150 subjects of age and sex matched, not satisfying International Diabetes Federation (IDF) criteria of metabolic syndrome were selected for the study as control. A deficiency in $25(\mathrm{OH}) \mathrm{D}$ levels were considered as below $20 \mathrm{ng} / \mathrm{ml}$, insufficiency if the levels were between 20 to $30 \mathrm{ng} / \mathrm{ml}$ and normal values were considered as above $30 \mathrm{ng} / \mathrm{ml}^{(16)}$. This was a prospective study conducted over the period of 24 months.
Inclusion criteria- Age :>20 years; both gender satisfying IDF Criteria for metabolic syndromewaist circumference $>90 \mathrm{~cm}$ in men and $>80 \mathrm{~cm}$ in women; plus two or more of the following: (1) hypertriglyceridemia fasting triglyceride $\geq 150$ $\mathrm{mg} / \mathrm{dL}(1.695 \mathrm{mmol} / \mathrm{L})$ or specific treatment for this lipid abnormality, (2) HDL cholesterol < 40 $\mathrm{mg} / \mathrm{dL}(1.036 \mathrm{mmol} / \mathrm{L})$ in men and $<50 \mathrm{mg} / \mathrm{dL}$ $(1.295 \mathrm{mmol} / \mathrm{L})$ in women or specific treatment for this lipid abnormality, (3) Blood pressure $\geq 130 / 85 \mathrm{mmHg}$ or treatment of previously diagnosed hypertension, (4) high fasting serum glucose $\geq 100 \mathrm{mg} / \mathrm{dL}(5.6 \mathrm{mmol} / \mathrm{L})$ or previously diagnosed type 2 diabetes.

Exclusion criteria- Patients with Nephrotic syndrme, Coronary heart disease, Pregnancy, Cerebrovascular disease, chronic renal failure, polycystic ovarian disease were excluded from the study.

Investigations- Complete haemogram, Urine routine and microscopic examination, Blood glucose levels -fasting and post prandial, Kidney function test, Lipid profile including serum triglycerides, serum cholesterol, HDL, LDL, vitamin-D levels. Collection of sample was done under strict aseptic precautions without using tourniquet; venous blood sample was collected from both cases and controls. Morning fasting blood sample was obtained and immediately sent to the laboratory for evaluation. $2 \mathrm{ml}$ blood was allowed to clot and then centrifuged to separate serum. This serum was used to estimate the level of 25-hydroxy vitamin-D using ELISA method [25-OH vitamin-D ELISA kit DL. D Dignostika GMBH, EA 300/96 kit].

\section{Statistical Analysis}

The statistical analysis was performed using statistical package for social sciences (SPSS) version 17 . The mean, standard deviation, median and ranges was calculated for continuous variables whereas proportion and frequency tables were used to summarize categorical variables. Chi square test and fisher exact test was used to compare categorical variables. Logistic regression 
has been performed taking outcome as dependent variables and laboratory parameters as independent variables to calculate the association between these variables. The level of significance was considered as $\mathrm{p}<0.05$.

\section{Results}

This study was performed on 150 cases and an equal numbers of controls to find the role of vitamin-D in metabolic syndrome, to find the prevalence of vitamin-D deficiency in cases of metabolic syndrome and to co-relate the parameters of metabolic syndrome which are maximally affected by vitamin-D deficiency.

\section{Cases}

Prevalence of Vitamin-D deficiency-Out of 150 cases in this study vitamin-D deficiency was seen in $90(60 \%)$ cases whereas only $30(20 \%)$ cases were adequate in vitamin-D stores. Rest 30(20\%) cases had an insufficient amount of vitamin D. Out of 78 males, vitamin-D deficiency was seen in 51 males and Out of 72 females vitamin-D deficiency was seen in 39 females. However it was not significant statistically (P-value $=.4197$ ). Out of 63 patients with raised blood pressures, only 36 cases had vitamin-D deficiency while 54 cases of vitamin-D deficiency had a normal blood pressure. It was not statistically significant. (Pvalue $=.7258)$. Out of 120 cases with high FBS, 84 cases had vitamin-D deficiency while only 6 cases with vitamin-D deficiency had normal fasting blood sugars. This was statistically significant. (P-value=.0096). Out of 90 cases with vitamin-D deficiency 72 cases had a higher triglyceride levels whereas only 18 cases with
vitamin-D deficiency had a normal triglyceride levels but it was not statistically significant.(Pvalue $=.3535$ ). Out of 90 cases with vitamin-D deficiency 51 cases had a lower HDL levels while 39 cases with vitamin-D deficiency had normal HDL levels, but this was not significant statistically. (P-value=.4197). It was observed that BMI was raised in all the 150 cases. Out of these $90(60 \%)$ cases had a vitamin-D deficiency while 30(20\%) cases had an insufficient levels of vitamin d. Maximum number of cases in this study were Housewives (32\%) followed by teachers and working women (12\% each). The minimum numbers of cases in this study were students and business women. Maximum prevalence of vitamin-D deficiency was seen in housewives and working women $20 \%$ each which is probably explained by their relative more indoor stay as compared to other subsets of population. It was statistically significant (Pvalue $=.0294$ )

Controls- Out of 33 controls with a raised fasting blood sugar levels, no control had vitamin-D deficiency. All the vitamin-D deficient controls had a normal fasting blood sugar values. No vitamin-D deficient controls had a raised blood pressure. Out of the controls with a raised triglyceride levels only $16 \%$ controls were deficient in vitamin-D which was not significant statistically. Out of the controls with a reduced HDL levels none of them were deficient in vitamin d.

Comparison between case and controls- It was seen that $60 \%$ of the cases were deficient in vitamin-D while only $10 \%$ of the controls were deficient in vitamin D. (Table3)

Table-1: Depicts the comparison of baseline parameters between the case and the controls

\begin{tabular}{|c|c|c|c|c|c|}
\hline Parameters & Group & $\mathbf{N}$ & Mean & Std. Deviation & Std. Error Mean \\
\hline \multirow[t]{2}{*}{ Systolic BP } & Case & 150 & 131.38 & 7.128 & 1.008 \\
\hline & Control & 150 & 122.00 & 5.511 & .779 \\
\hline \multirow[t]{2}{*}{ Diastolic BP } & Case & 150 & 83.30 & 6.042 & .854 \\
\hline & Control & 150 & 76.96 & 4.375 & .619 \\
\hline \multirow[t]{2}{*}{ FBS } & Case & 150 & 117.54 & 17.934 & 2.536 \\
\hline & Control & 150 & 108.46 & 112.993 & 15.980 \\
\hline \multirow[t]{2}{*}{ S.TG } & Case & 150 & 182.76 & 31.983 & 4.523 \\
\hline & Control & 150 & 134.22 & 15.064 & 2.130 \\
\hline \multirow[t]{2}{*}{ HDL } & Case & 150 & 40.84 & 8.975 & 1.269 \\
\hline & Control & 150 & 50.48 & 5.084 & .719 \\
\hline
\end{tabular}




\section{JMSCR Vol||06||Issue||03||Page 676-681||March}

Table 2: depicts the association of number of metabolic syndrome components with Vitamin-D mean values in vitamin-D deficient cases. As the number of components increases the mean value of vitamin-D reduces.

\begin{tabular}{|lccc|} 
Components & $\begin{array}{c}\text { Total cases } \\
(\mathbf{n}=\mathbf{1 5 0})\end{array}$ & $\begin{array}{c}\text { Number of vitamin-D } \\
\text { Deficient cases }\end{array}$ & $\begin{array}{c}\text { Mean Vitamin-D } \\
\text { level }\end{array}$ \\
\hline $\mathbf{3}$ & $\mathbf{7 2}$ & $\mathbf{3 9}$ & $\mathbf{2 2 . 7 7}$ \\
\hline $\mathbf{4}$ & $\mathbf{5 1}$ & $\mathbf{3 3}$ & $\mathbf{1 7 . 1 8}$ \\
\hline $\mathbf{5}$ & $\mathbf{2 7}$ & $\mathbf{1 8}$ & $\mathbf{1 6 . 9 8}$ \\
\hline
\end{tabular}

Table 3: Reflects the prevalence of various parameters of metabolic syndrome along with respective vitamin-D value.

\begin{tabular}{|c|c|c|c|c|}
\hline Parameters & Controls $(\mathrm{n}=\mathbf{1 5 0})$ & Controls \%age & Cases $(n=150)$ & $\begin{array}{l}\text { Cases } \\
\% \text { age }\end{array}$ \\
\hline VitaminD<20 (ng/ml) & 15 & 10 & 90 & 60 \\
\hline $20-29(\mathrm{ng} / \mathrm{ml})$ & 36 & 24 & 30 & 20 \\
\hline$>30(\mathrm{ng} / \mathrm{ml})$ & 99 & 66 & 30 & 20 \\
\hline Waist circum: Normal & 144 & 96 & 150 & 100 \\
\hline Raised & 6 & 4 & $\mathbf{0}$ & $\mathbf{0}$ \\
\hline FBS: Normal(100mg/dl) & 117 & 78 & 30 & 20 \\
\hline Raised & 33 & 33 & 120 & 80 \\
\hline BP: Normal $(<130 / 85)$ & 147 & 98 & 87 & 58 \\
\hline Raised (>130/85) & 3 & 2 & 63 & 42 \\
\hline S.TAG: Normal(<150) & 132 & 88 & 24 & 16 \\
\hline Raised $(>150)$ & 18 & 12 & 126 & 84 \\
\hline HDL: $\operatorname{Normal}(m=40, f=50)$ & 132 & 88 & 72 & 48 \\
\hline Raised & 12 & 12 & 78 & 52 \\
\hline Sex: male & 78 & 52 & 78 & 52 \\
\hline Female & 72 & 48 & 72 & 48 \\
\hline Age: $20-29$ yrs & 15 & 10 & 15 & 10 \\
\hline 30-39 yrs & 96 & 64 & 96 & 64 \\
\hline $40-49$ yrs & 27 & 18 & 27 & 18 \\
\hline & 12 & 8 & 12 & 8 \\
\hline
\end{tabular}

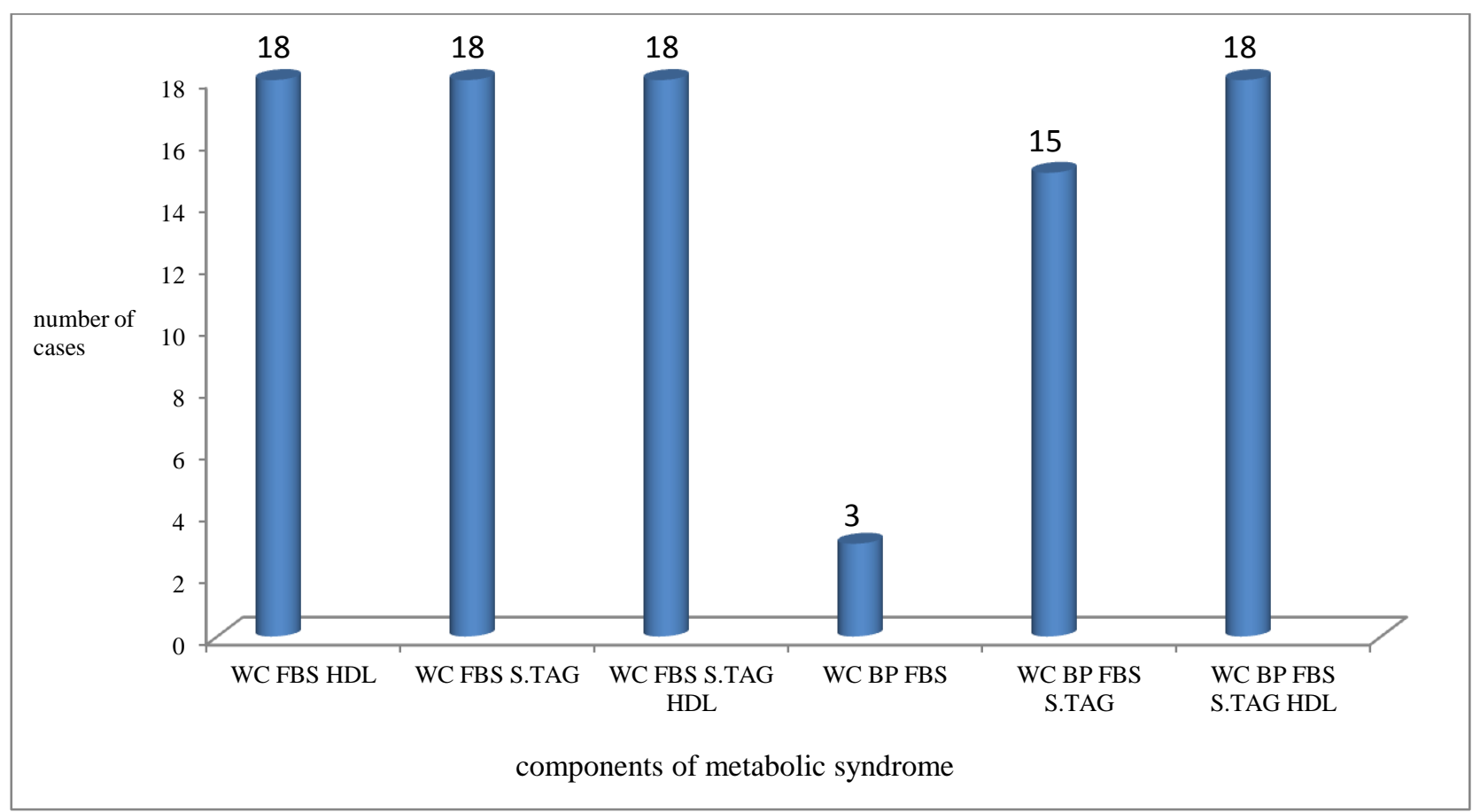

Figure: 1 Graph representing correlation of number of cases with Vitamin-D deficiency and component of metabolic syndrome .It depicts the patients with only WC,BP and FBS as their components of metabolic syndrome had least vitamin- D deficiency compared to others.

WC-Waist circumference, FBS- fasting blood sugar, HDL- High density lipoprotein, S.TG- Serum triglyceride, BP- Blood pressure 


\section{Discussion}

Metabolic syndrome is a pro-inflammatory state seems to have a determining role in the further morbidities ascribed to $\mathrm{MeS}$, including diabetes and cardiovascular disease . Considering the antiinflammatory effects of cholecalciferol, poor vitamin-D status may contribute to the systemic inflammation often seen in obesity, thus favoring other metabolic derangements like insulin resistance and raised blood glucose. It has been hypothesized that low serum vitamin-D could play a significant role in the pathogenesis of glucose intolerance and type 2 diabetes. It is worth noting that vitamin-D is a fat soluble vitamin. Thus, increasing adiposity may serve as a repository (or metabolic sink) for vitamin-D storage.

It was seen that vitamin-D levels decreased as the cases had more than 3 components of metabolic syndrome with mean vitamin-D levels. The mean vitamin-D levels showed an inverse relationship with number of components of metabolic syndrome in an individual. It was clearly seen that cases had a higher mean values of systolic blood pressure, diastolic blood pressure, fasting blood sugars, s. triglyceride levels as compared to the controls .Cases had a lower mean HDL levels as compared to the controls in whom the mean HDL levels were higher. In this study we found a significant inverse association between mean vitamin-D levels and cases of metabolic syndrome those had components including waist circumference, hyperglycemia and hypertriglyceridemia (Table 2) .

It was seen that most of cases had higher fasting blood sugar level and vitamin-D level was also deficient in cases those had higher FBS and this was statistically significant. Similar significant association was found in studies conducted by Ken 2004, Alvarez \& Ashraf \& Chiu et al ${ }^{(11)}$. We also found that most of cases also had higher serum triglyceride level and vitamin-D level was also deficient in these cases but this was not statistically significant. In this study blood pressure had weakest association with metabolic syndrome and vitamin-D level. The limitations of our study involved a small sample size and crosssectional nature of the study.

\section{Conclusion}

We therefore conclude that serum vitamin-D level decreased as cases has more than three components and shows inverse relationship with increasing number of components of metabolic syndrome. Vitamin-D replacement therapy should be considered for metabolic syndrome patients in view of increase prevalence of vitamin-D deficiency.

\section{References}

1. Third Report of the National Cholesterol Education Program (NCEP) expert panel on detection, evaluation, and treatment of high blood cholesterol in adults (Adult Treatment Panel III) final report. Circulation 2002;106(25):3143-3421.

2. Despres JP, Lemieux I, Bergeron J, et al. Abdominal obesity and themetabolic syndrome: contribution to global cardio metabolic risk. Arterioscler Thromb Vasc Biol 2008;28(6):1039-1049.

3. Boucher BJ. Inadequate vitamin-D status: does it contribute to the disorders comprising syndrome " $\mathrm{X}$ "? Br J Nutr 1998;79(4):315-327.

4. Elks CM, Francis J. Central adiposity, systemic inflammation, and the metabolic syndrome. Curr Hypertens Rep 2010;12 (2):99-104.

5. Azadbakht L., Mirmiran P., Esmaillzageh A., Azizi F. Dairy consumption is inversely associated with the prevalence the metabolic syndrome in Tehranian adults. Am J clin Nutr 2005; 82: 523-530.

6. Isaica G., Giorgino R., Adami S. High prevalence of hypovitaminosis $\mathrm{D}$ in female type 2 diabetic population ( Letter). Diabetes care 2001;24: 1496.

7. Maghbooli Z., Hossein-Nezhad A., Karimi, F., Shafaei A.R., Larijani B. Correlation between vitamin D3 
deficiency and insulin resistance in pregnancy. Diabetes Metab Res Rev 2008;24:27-32.

8. Scragg R., Sowers M., Bell, C. Serum 25hydroxyvitamin D, Diabetes, and ethnicity in third national Health and Nutrition Examination scurvy. Diabetes care 2004;27:2813-2818.

9. Maghbooli Z., Hossein- Nezhad A., Khoshniat. M., Adibi H.,Mohamadzadesh, N., Larijani B. A study of bone mineral density in diabetic patients, Iranian journal of public Health 2007;Suppl: 37-44.

10. Maghbooli Z., Hossein-Nezhad A., Shafaei A.R., Karimi F., Madani F.S.,Larjani, B. Vitamin-D status in mothers and their newborns in Iran. BMC Pregnancy and childbirth 2007; 7: 1-6

11. Chiu K.C., Chu A., Go V.L., Saad M.F. Hypovitaminosis D is associated with insulin resistance and beta cell dysfunction. Am J clin Nutr 2004; 79:820-825

12. Baynes K.C., Boucher B.j., Feskens E.J., Kromhout D. vitamin D, glucose tolerance and insulinemia in elderly men. Diabetologia 1997; 40:344-347

13. Boucher B.J., MannanN., Nooonan K., Hales, C.N., EvansS.J. Glucose intolerance and impairment of insulin secretion in relation to vitamin-D deficiency in east London Asians. Diabetologia 1995; 38: 1239-1245

14. Llind L., Hanni A., Lithell H., Hvarfner A., Sorenson O.H., Ljanghall S. Vitamin$\mathrm{D}$ is related to blood pressure and other cardio vascular risk factors in middle aged men. Am J Hypertens 1995; 8:894-901

15. Ortlepp J.R., Metrikat J., Albrecht M., KorffA., HarnathP., HoffmannR. The vitamin-D receptor gene variant and physical activity predicts fasting glucose levels in healthy young men. Diabet Med 2003; 20: 451-454. 